\title{
Structural analysis of Fe-Mn-O nanoparticles in glass ceramics by Small Angle Scattering
}

\author{
Vikram Singh Raghuwanshi, ${ }^{*, a}$ Ruzha Harizanova, ${ }^{\mathrm{b}}$ Dragomir Tatchev, ${ }^{\mathrm{c}}$ Armin \\ Hoell, ${ }^{1}$ Christian Rüssel ${ }^{\mathrm{d}}$
}

${ }^{a}$ Helmholtz Zentrum Berlin für Materialien und Energie, Hahn-Meitner Platz 1, D-14109, Berlin, Germany

${ }^{b}$ University of Chemical Technology and Metallurgy, 8 Kl. Ohridski Blvd, 1756 Sofia, Bulgaria

${ }^{c}$ Institute of Physical Chemistry, Bulgarian Academy of Sciences, Acad. G. Bonchev Str. Bl. 11, 1113 Sofia, Bulgaria

${ }^{d}$ Friedrich Schiller University, Fraunhoferstr. 6, 07743 Jena, Germany

\begin{abstract}
Magnetic nanocrystals containing $\mathrm{Fe}$ and $\mathrm{Mn}$ were obtained by annealing of silicate glasses with the composition $13.6 \mathrm{Na}_{2} \mathrm{O}-62.9 \mathrm{SiO}_{2}-8.5 \mathrm{MnO}-15.0 \mathrm{Fe}_{2} \mathrm{O}_{3-\mathrm{x}}(\mathrm{mol} \%)$ at 580 ${ }^{\circ} \mathrm{C}$ for different periods of time. Here, we present Small Angle Neutron Scattering using Polarized neutrons (SANSPOL) and Anomalous Small Angle X-ray Scattering (ASAXS) investigation on these glass ceramic samples. Analysis of scattering data from both methods reveals the formation of spherical core-shell type of nanoparticles with mean sizes between 10 $\mathrm{nm}$ and $100 \mathrm{~nm}$. ASAXS investigation shows the particles have higher concentration of iron atoms and the shell like region surrounding the particles is enriched in $\mathrm{SiO}_{2}$. SANSPOL investigation shows the particles are found to be magnetic and are surrounded by a nonmagnetic shell-like region.
\end{abstract}

Keywords: ASAXS, SANSPOL, Glass ceramics, Magnetic nanoparticles

*Corresponding author, E-mail: vikram.raghuwanshi@helmholtz-berlin.de Introduction

Oxide glass ceramics containing magnetic nanocrystals with sizes ranging from $1 \mathrm{~nm}$ to $100 \mathrm{~nm}$ show peculiar magnetic and electric properties and in the future can find many applications, e.g. in the fields of medical, information technology, telecommunication, as 
ferrofluids and in electronics [1-5]. They can be used as parts of cooling or shielding devices for electronic components [6]. As a part of ferrofluids, they have applications in magnetic and biomagnetic sensors, improving the sound quality of loudspeakers and in magnetic resonance imaging (MRI). [7, 8]. The functionality of the material depends on size, shape, volume fraction, distribution and composition of the precipitated crystalline phase and the interaction of crystalline phase with the neighboring nanocrystals as well as with the host matrix [9].

In the present work, the glass ceramic under study has the composition $13.6 \mathrm{Na}_{2} \mathrm{O}$ $62.9 \mathrm{SiO}_{2}-8.5 \mathrm{MnO}-15.0 \mathrm{Fe}_{2} \mathrm{O}_{3-\mathrm{x}}(\mathrm{mol} \%)$. The as prepared glasses were annealed at $580{ }^{\circ} \mathrm{C}$ for different times varying from $10 \mathrm{~min}$ to $60 \mathrm{~min}$, which lead to the precipitation of the magnetic nanocrystals as shown previously $[10,11]$. Earlier, for the same series of samples annealed at $550{ }^{\circ} \mathrm{C}$, it was shown by ASAXS [12] that the magnetite-jacobsite containing particles are surrounded by shells depleted in Fe and $\mathrm{Mn}$ which are expected to be non-magnetic. The main goal of this work is to estimate the effect of heat treatment on the structure parameters and to evaluate the magnetic behaviour of the nanoparticles embedded in the studied glass ceramic material. The SANSPOL technique was applied in order to reveal the structure parameters and distribution of nanoparticles in the glass matrix. Moreover, this technique allows studying the magnetic nature of the particles. SANS measurements with polarized neutrons were performed at the D22 SANS instrument at the Institute Laue-Langevin (ILL), Grenoble France [13]. ASAXS technique was applied to reveal the distribution of particular element in the nanoparticles and to estimate quantitatively the density, volume fraction and percentage of $\mathrm{Fe}$ atoms in the respective magnetic nanoparticles. The ASAXS experiments were performed near the X-ray K absorption edges of Fe $(7112 \mathrm{eV})$ and of Mn $(6539 \mathrm{eV})$ using the 7T-MPW-SAXS beamline at the synchrotron BESSY II at Helmholtz-Zentrum Berlin (HZB) [14].

\section{Theory}

\section{SAS/ASAXS}

Small angle scattering (SAS) is an analytical technique to determine structure parameters of particles in the size range from about $1 \mathrm{~nm}$ to $100 \mathrm{~nm}$ [15-17]. In small angle scattering experiments, the spatial correlations in the scattering densities present in the sample are measured. The differential scattering cross section of a system of spherical particles with the number density distribution $N(r)$ can be described as:

$$
\frac{d \sigma}{d \Omega}(\vec{q})=\int_{0}^{\infty} N(r) V_{p}(r)^{2}|F(q, r, \Delta \eta(E))|^{2} d r+B k g
$$


where $\Delta \eta=\eta_{\text {particle }}-\eta_{\text {matrix }}$ is the electron density difference between the particles and the remaining matrix. The volume of the particle is given by $V_{p}(r)$. Here $|\vec{q}|$ is the scattering vector, which is related with the scattering angle of $2 \theta$ as follows:

$$
|\vec{q}|=q=\frac{4 \pi \sin \theta}{\lambda}
$$

where $\lambda$ is the wavelength of the X-rays or neutrons. In equation (1), $F(q, r, \Delta \eta(E))$ is the form factor that accounts for the shape and the size of the particles. For a homogeneous sphere with radius $r$, the form factor is defined as [18,19]:

$F(q, r, \Delta \eta)=3 \Delta \eta \frac{\sin (q r)-(q r) \cos (q r)}{(q r)^{3}}$

Subtracting the form factor of the inner sphere from the outer sphere provides the form factor for a spherical core-shell particle given as:

$F\left(q, r, \Delta \eta_{\text {shell }}, \mu\right)=F\left(q, r, \Delta \eta_{\text {shell }}\right)-F\left(q, v r, \Delta \eta_{\text {shell }}(1-\mu)\right)$

where $\Delta \eta_{\text {shell }}$ is the scattering contrast for the shell and $\mu \cdot \Delta \eta_{\text {shell }}$ is the scattering contrast for the core, both contrasts are with respect to the surrounding matrix. $r$ is the distance from the particle centre till the end of the shell and $v r(0<v<1)$ is the radius of the core.

In equation (1), a constant scattering background coming from fluorescence and resonant Raman scattering is added.

In ASAXS, the variation of the scattering power of specific element helps in determining the quantitative information on the distribution of that specific element in the nanoparticles and the respective phase compositions as well as the volume fractions $[12,21,22]$. In ASAXS measurements, the energy dependent atomic scattering amplitudes play an important role. When the photon energy approaches the absorption edge of an element, the atomic scattering amplitude varies significantly because of electronic excitations. The atomic scattering factor is given as:

$f(E)=f 0+f^{\prime}(E)+i f^{\prime \prime}(E)$

Here, $f_{0}+f^{\prime}(E)$ is the real and $f^{\prime \prime}(E)$ is the imaginary part of the scattering amplitude. $f^{\prime}(E)$ and $f^{\prime \prime}(E)$ are related to each other by Kramers-Kronig relation. The values of the scattering amplitudes $f^{\prime}(E)$ and $f^{\prime \prime}(E)$ are taken from the Cromer and Liberman calculations [23].

Quantitative information about the structure parameters of the core, shell and the remaining matrix were obtained by using two separate fit routines. First, the experimental 
relative contrasts were evaluated by simultaneous fitting of ASAXS curves by using the software SASfit [24]. Second, the experimental relative contrasts, estimated by the first fitting routine, were further fitted with the theoretically calculated contrasts in a separate MATLAB routine in order to get the phase composition as reported earlier [12,22].

Contribution of a particular element (resonant atoms) to the scattering intensity can be separated from the total scattering amplitude by evaluating the resonant curve using the Stuhrmann equation $[25,26]$

$$
I(q, E)=F_{0}(q)+f^{\prime}(E) F_{0 R}(q)+\left[f^{\prime}(E)^{2}+f^{\prime \prime}(E)^{2}\right] F_{R}(q)
$$

Here, $F_{0}(q)$ is the normal SAXS term (far from the absorption edge), $F_{0 R}(q)$ is the scattering cross term and $F_{R}(q)$ is the resonant scattering term. Measuring the scattering intensities at three different energies or more can form a set of linear equations by using equation (6). Solving the set of equations give the value of variables, $F_{0}(q), F_{0 R}(q)$ and $F_{R}(q)$.

\section{SANSPOL}

In SANSPOL experiments, apart of the nuclear interaction there is a contribution of the magnetic moment of the atoms that come from its interaction with the magnetic moments of the neutrons [27-31]. During measurements a constant magnetic field in the direction perpendicular to the direction of the incident neutron beam is applied to the sample. The neutron spins are either parallel (denoted by -) or antiparallel (denoted by + ) to the applied magnetic field direction, depending on the polarization state of the incoming neutrons. The scattering cross sections for parallel and antiparallel polarization state of neutrons are given by $[32,33]$ :

$$
\begin{aligned}
\frac{d \sigma}{d \Omega}(q, \psi)^{-} & =F_{N}^{2}\left(q, r, \Delta \eta_{\text {nucl }}\right)+2 F_{M}^{2}\left(q, r, \Delta \eta_{\text {magn }}\right) \frac{L(x)}{x}+\left\{F_{M}^{2}\left(q, r, \Delta \eta_{\text {magn }}\right)\left[1-3 \frac{L(x)}{x}\right]\right. \\
& \left.+2 P(2 \varepsilon-1) F_{N}\left(q, r, \Delta \eta_{\text {nucl }}\right) F_{M}\left(q, r, \Delta \eta_{\text {magn }}\right)\right\} \sin ^{2} \psi \\
\frac{d \sigma}{d \Omega}(q, \psi)^{+}= & F_{N}^{2}\left(q, r, \Delta \eta_{\text {nucl }}\right)+2 F_{M}^{2}\left(q, r, \Delta \eta_{\text {magn }}\right) \frac{L(x)}{x}+\left\{F_{M}^{2}\left(q, r, \Delta \eta_{\text {magn }}\right)\left[1-3 \frac{L(x)}{x}\right]\right. \\
& \left.-2 P F_{N}\left(q, r, \Delta \eta_{\text {nucl }}\right) F_{M}\left(q, r, \Delta \eta_{\text {magn }}\right)\right) \sin ^{2} \psi
\end{aligned}
$$

where $\psi$ is the angle between the direction of the magnetic field and the momentum transfer vector $q . F_{N}\left(q, r, \Delta \eta_{n u c l}\right)$ and $F_{M}\left(q, r, \Delta \eta_{\text {magn }}\right)$ are the nuclear and magnetic form factors of the nanoparticles. $\Delta \eta_{\text {nucl }}$ is the nuclear contrast which is the difference between the neutron 
scattering length densities of the different phases present in the sample. The scattering length density is given as:

$$
\eta_{i}=\frac{\sum_{i=1}^{N} c_{i} b_{i}}{\sum_{i=1}^{N} c_{i} M_{i}}
$$

and $\Delta \eta_{\text {magn }}$ is the magnetic contrast which is the difference between the magnetic scattering length densities of the different phases present in the sample.

$\eta_{\text {magn }}=\frac{\sum_{i=1}^{N} c_{i} m_{i}}{\sum_{i=1}^{N} c_{i} M_{i}}$

In equations (9) and (10), $c_{i}$ is the concentration, $b_{i}$ is the nuclear scattering length, $m_{i}$ is the magnetization perpendicular to the momentum vector $q$ and $M_{i}$ is the molar mass of the $i^{\text {th }}$ constituent of the phase. $P$ in equations (7) and (8) is the polarization of the neutron beam and is represented as:

$P=\frac{N^{+}-N^{-}}{N^{+}+N^{-}}$

where $N^{+}$is the number of neutrons with spin anti-parallel and $N^{-}$spin parallel to the applied magnetic field direction $H$. The efficiency of the spin flipper is represented by $\epsilon$. In equations (7) and (8), $L(x)=\operatorname{coth}(x)-1 / x$ is the Langevin function with $x=m v H /\left(K_{B} T\right)$ here $H$ is the applied magnetic field, $m$ is the magnetization and $v$ is the volume of the particle. $K_{B} T$ is the thermal energy at temperature $T$ and $K_{B}$ is the Boltzmann's constant.

By using equations (7) and (8), the scattering cross section for non-polarized beam can be determined as:

$\frac{d \sigma}{d \Omega}(q, \psi)=\frac{\frac{d \sigma}{d \Omega}(q, \psi)^{+}+\frac{d \sigma}{d \Omega}(q, \psi)^{-}}{2}$

From the above equation (12), one can see that, if the direction of the vector $q$ is parallel to the magnetization direction $\left(\psi=0^{\circ}\right)$, then the magnetic scattering has no contribution, while for $q$ perpendicular to the magnetization direction $\left(\psi=90^{\circ}\right)$ magnetic scattering could have a significant contribution to the total scattering. For $\psi=0^{\circ}$ in equation (12), the nuclear scattering cross section is given as: 
$\left(\frac{d \sigma}{d \Omega}(\vec{q})\right)_{\text {nuclear }}=\left|F_{N}\left(q, r, \Delta \eta_{\text {nucl }}\right)\right|^{2}$

Putting $\psi=90^{\circ}$, subtracting the nuclear scattering cross section from equation (12) and accounting that spin flipper efficiency $\varepsilon \cong 1$, one obtains the magnetic scattering cross section as:

$\left(\frac{d \sigma}{d \Omega}(\vec{q})\right)_{\text {magnetic }}=\left|F_{M}\left(q, r, \Delta \eta_{\text {magn }}\right)\right|^{2}-2 P(1-\varepsilon) F_{M}\left(q, r, \Delta \eta_{\text {magn }}\right) F_{N}\left(q, r, \Delta \eta_{\text {nucl }}\right)$

For strong magnetic field and large particle sizes, magnetic moments are well aligned in the direction of the field and the Langevin function $L(x)$ in the equations (7) and (8), vanishes because at larger $x(x \rightarrow \infty), L(x) \rightarrow 1$ and $L(x) / x \rightarrow 0$. In the investigated samples, particles are fully magnetized under the applied field of 1.5 Tesla.

\section{Experimental}

The base glass used in our study has the composition $13.6 \mathrm{Na}_{2} \mathrm{O}-62.9 \mathrm{SiO}_{2}-8.5 \mathrm{MnO}-$ $15.0 \mathrm{Fe}_{2} \mathrm{O}_{3-\mathrm{x}}\left(\mathrm{mol} \%\right.$ ). It was produced by using reagent grade compounds: $\mathrm{Na}_{2} \mathrm{CO}_{3}, \mathrm{MnCO}_{3}$, $\mathrm{SiO}_{2}$ and $\mathrm{FeC}_{2} \mathrm{O}_{4} \cdot 2 \mathrm{H}_{2} \mathrm{O}$. The batches $(100 \mathrm{~g})$ were melted and homogenized in $\mathrm{SiO}_{2}$-crucibles using a $\mathrm{MoSi}_{2}$-furnace with temperatures in the range from 1400 to $1450{ }^{\circ} \mathrm{C}$ (kept for $1.5 \mathrm{~h}$ in air). One part of the melt was quenched on a Cu-block (cooling rate $300 \mathrm{~K} / \mathrm{min}$ ), while the rest was casted into a pre-heated graphite mould (cooling rate $200 \mathrm{~K} / \mathrm{min}$ ). All cast glasses were further transferred to a muffle furnace and kept at $480{ }^{\circ} \mathrm{C}$ for $10 \mathrm{~min}$. Then, the furnace was switched off and the samples were cooled down to room temperature. Finally the samples were polished to thickness between $800 \mu \mathrm{m}$ to $2000 \mu \mathrm{m}$ for the SANSPOL experiments and the samples with thickness of $150 \mu \mathrm{m}$ were prepared for the ASAXS measurements in order to achieve optimal transmission. Later on, pieces of the as prepared glass samples were annealed at $580{ }^{\circ} \mathrm{C}$ for different time intervals in the range from $10 \mathrm{~min}$ to $60 \mathrm{~min}$.

The SANS experiments were performed at the D22 SANS instrument at the Institute LaueLangevin (ILL), Grenoble France [13]. The incident neutrons were monochromatized by means of a velocity selector. Neutrons with a wavelength of $0.6 \mathrm{~nm}$ were selected. During the measurement, a static magnetic field of 1.5 Tesla was applied in horizontal direction and perpendicular to the direction of the incident neutron beam. A polarizer was used to polarize the beam and guided through the collimation system. A radio frequency spin flipper was used in two possible states ON or OFF to flip the spin direction of the neutrons. Depending on its state, the flipper switches the spin of the polarized neutrons either parallel or antiparallel to 
the applied magnetic field direction. Each sample was measured twice, once with the flipper ON and once with the flipper OFF state. The scattered neutrons were collected using a multitube detector. The samples were measured at three different sample detector distances $(2,8$ and $17.6 \mathrm{~m}$ ) to achieve a wide $q$ range. The raw data were corrected for background, transmission, scaled to differential scattering cross section per unit volume and then anisotropic averaging was performed to obtain the final scattering curves. The raw data reduction was done by using the software GRASP [13].

The ASAXS experiments were carried out by choosing five different energies near and below to the $\mathrm{K}$ absorption edges of Fe $(7112 \mathrm{eV})$ and $\mathrm{Mn}(6539 \mathrm{eV})$ at the 7T-MPW SAXS beamline at BESSY II. The photons scattered by the sample were collected by using multiwire proportional counter gas filled area detector with pixel size of $207 \mu \mathrm{m}$. While measuring, the detector was placed at two different positions, far from the samples $(3745 \mathrm{~mm})$ and close to the samples $(700 \mathrm{~mm})$ in order to achieve wide $q$ range. The scattering images were corrected for transmission, photon flux, dead-time of the detector electronics and sensitivity of detector pixels and finally after circular averaging the scattering curves were obtained. A pre-calibrated glassy carbon standard sample was used to scale the scattering curves to differential scattering cross sections per unit volume. A silver behenate sample (peak at $\mathrm{q}=1.076 \mathrm{~nm}^{-1}$ ) was used to calibrate the $q$ scale. The data reduction was done by using the software SASREDTOOL [34].

\section{Results}

SANSPOL experiments were performed to obtain structure information of precipitated nanoparticles in glass ceramics with magnetic properties. This type of neutron scattering investigation allows to separate the nuclear scattering and magnetic scattering of the sample. Figure 1 shows the anisotropic scattering images of different polarization states of incoming neutrons for $(\mathrm{ON})$ and $(\mathrm{OFF})$ and the difference term (ON - OFF) for the sample annealed at $580{ }^{\circ} \mathrm{C}$ for $40 \mathrm{~min}$. The difference term shows the pronounced scattering in the direction perpendicular to the magnetic field, which reveals that the significant part of magnetic moments are aligned in the direction of the applied field. 

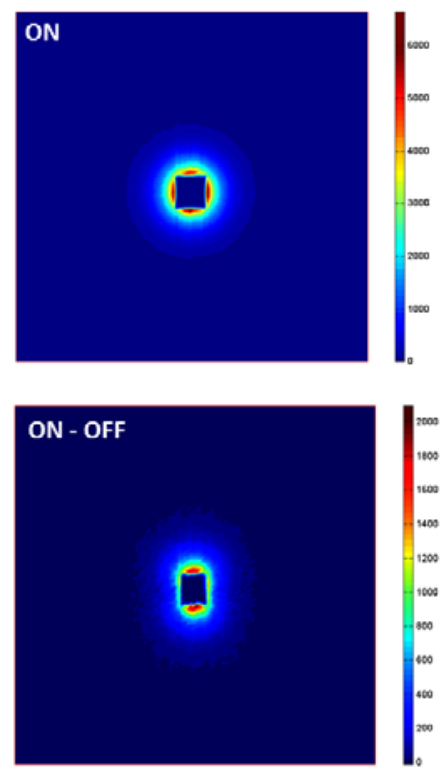

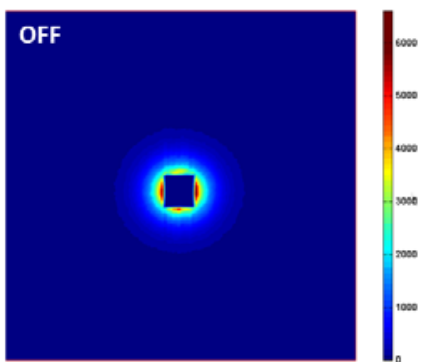

\section{Magnetic Field (H)}

$\mathrm{H} \rightarrow \mathbf{1 . 5}$ Tesla

Figure 1: 2D scattering intensity patterns from the polarized SANS experiments after data correction for the sample annealed at $580^{\circ} \mathrm{C}$ for $40 \mathrm{~min}$. The magnetic field of 1.5 Tesla is applied in horizontal direction perpendicular to the incident neutron beam. Intensities are measured under different polarization states of incident neutrons antiparallel $(O N)$ to the magnetic field, parallel $(O F F)$ to the magnetic field, and (ON - OFF) is the difference term of (ON) and (OFF)

Nuclear and magnetic scattering were separated by using the cosine on second power variation of the magnetic scattering intensity within a circle of constant $q$ around the centre of the detector image [13]. Separated nuclear and magnetic scattering curves for the sample annealed at $580{ }^{\circ} \mathrm{C}$ for $40 \mathrm{~min}$ are shown in Figure 2. The scattering curves are scaled to the differential scattering cross section per unit volume and plotted as a function of the momentum transfer vector $q$. Pronounced amount of magnetic scattering curve reveals the presence of a phase with strong magnetic properties.

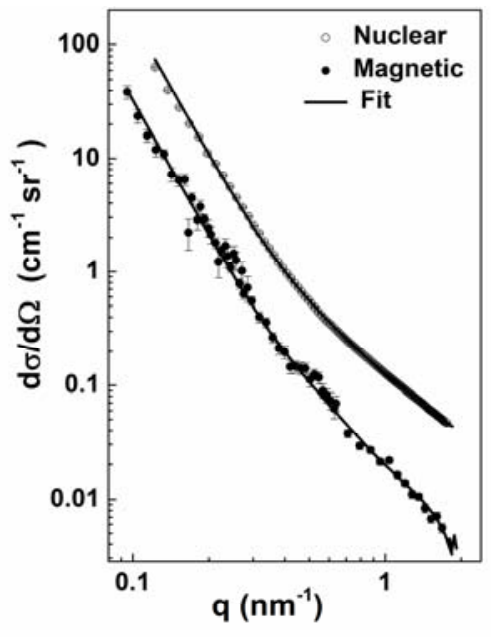

Figure 2: Separated nuclear and magnetic SANS curves for the sample annealed at $580{ }^{\circ} \mathrm{C}$ for 40 min. The nuclear curve is fitted with the spherical core-shell model and the magnetic curve is fitted with spherical model. Solid line shows the fit. 
Similarly, nuclear and magnetic scattering curves for the samples annealed at $580{ }^{\circ} \mathrm{C}$ for 20, 40 and 60 min are shown in Figures 3(a) and 3(b). It is seen that the intensity of the scattering curves for the samples annealed at 20,40 and 60 min increases with the annealing time. To extract the structure information, the scattering curves were fitted with equation (1), by using the software SASfit [24]. To fit any scattering curve, SASfit requires a pre-defined form factor and the shape of the size distribution.
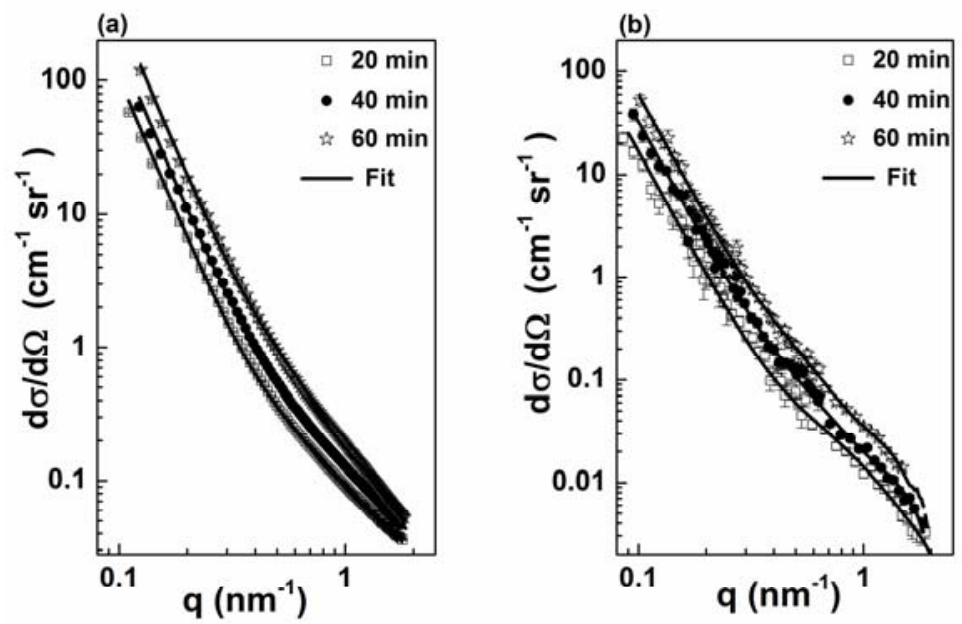

Figure 3: Nuclear and magnetic scattering curves for the sample annealed at $580{ }^{\circ} \mathrm{C}$ for different time periods. (a) Nuclear scattering curves are fitted with the spherical core-shell model. (b)

Magnetic scattering curves are fitted with the spherical model. Solid line shows the fit.

The nuclear scattering curves for the sample annealed at $580{ }^{\circ} \mathrm{C}$ for 20,40 and $60 \mathrm{~min}$ are fitted by assuming the spherical core-shell model shown in Figure 4 (Left) and the log normal distribution of particles. In Figure 4 (Right), a contrast variation profile is shown from the SANS curve fitting. The profile shows the electron density of the cores has higher and the shell has lower electron density with respect to the matrix. Moreover, the magnetic scattering curves are fitted by assuming a sphere model. Figure 3 shows the fit of all respective SANS curves. Resulting size parameters obtained after fitting of the SANS curves are shown in Table 1. 

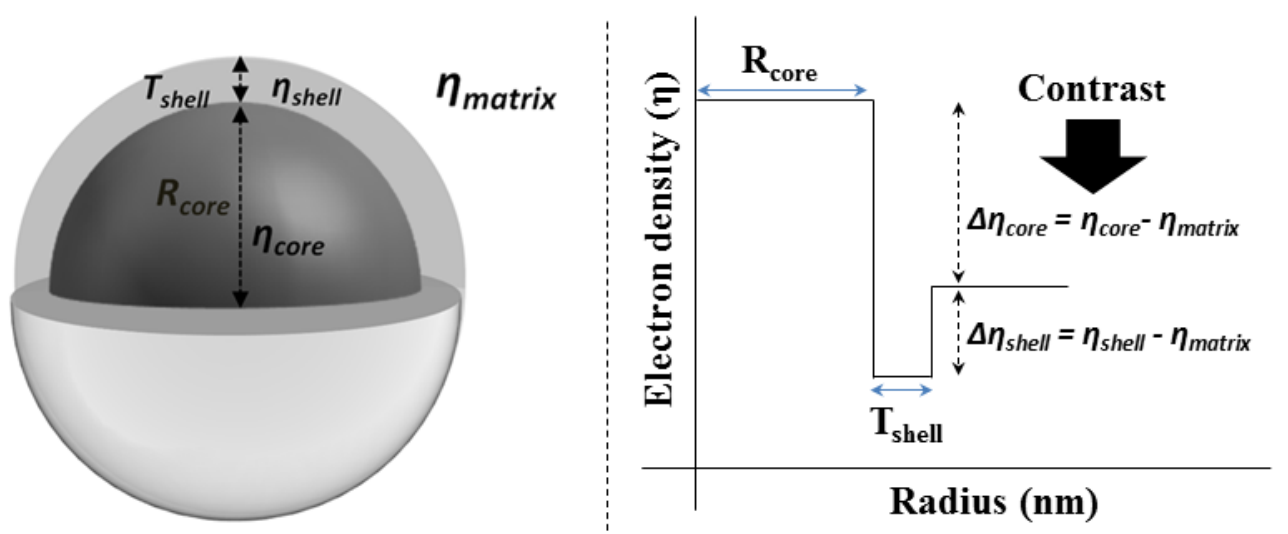

Figure 4: Sketch of the spherical core-shell model (left). Relative contrast profile to fit the SANS and ASAXS curves (right).

Table 1 Averaged size parameters calculated from fitting the nuclear and magnetic scattering curves as extracted from the polarized SANS experiment for the samples annealed at $580{ }^{\circ} \mathrm{C}$ for 20,40 and $60 \mathrm{~min}$.

\begin{tabular}{|c|c|c|c|}
\hline \multirow[t]{2}{*}{ Sample $580^{\circ} \mathrm{C}$} & Nuclear & Scattering & $\begin{array}{l}\text { Magnetic } \\
\text { Scattering }\end{array}$ \\
\hline & $\begin{array}{c}\text { Average } \\
\text { Particle Radius } \\
\text { (nm) }\end{array}$ & $\begin{array}{c}\text { Average Shell } \\
\text { Thickness } \\
\text { (nm) }\end{array}$ & $\begin{array}{c}\text { Average Magnetic } \\
\text { particle Radius } \\
(\mathrm{nm})\end{array}$ \\
\hline $20 \mathrm{~min}$ & $21.5 \pm 1.0$ & $1.5 \pm 0.3$ & $19.5 \pm 1.0$ \\
\hline $40 \mathrm{~min}$ & $29.3 \pm 1.0$ & $1.9 \pm 0.3$ & $30.0 \pm 1.0$ \\
\hline $60 \mathrm{~min}$ & $38.5 \pm 2.0$ & $2.4 \pm 0.3$ & $37.5 \pm 2.0$ \\
\hline
\end{tabular}

The parameters reveal growth of particles with the annealing time. Also the thickness of the shell increases with the annealing time. It is shown in Table 1 that, for nuclear scattering curves, the size of the core (particle) in the spherical core-shell model is comparable to the size of the sphere used in the sphere model for magnetic scattering curves within the experimental accuracy. This result reveals that the particles are magnetic in nature and they are surrounded by a non-magnetic shell-like region. The size distributions of particles evaluated by fitting of the nuclear and magnetic scattering are shown in Figure 5. 


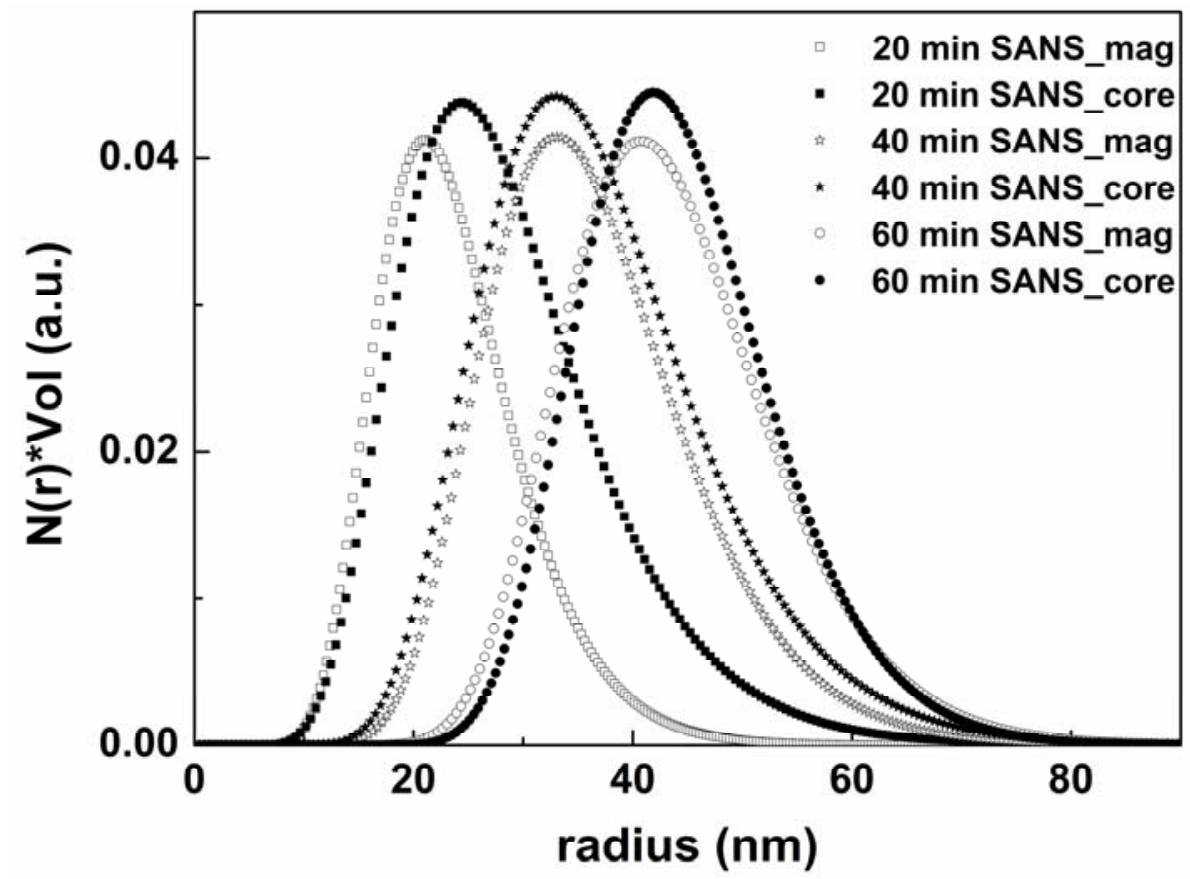

Figure 5: Comparison of log normal size distributions for the magnetic and nuclear scattering estimated by fitting the respective scattering curves.

Quantitative information about the composition and structure can be estimated by using the ASAXS technique [21]. Figures 6(a) and 6(b) show the ASAXS measurement at five different energies near the X-ray $\mathrm{K}$ absorption edges of Fe $(7112 \mathrm{eV})$ and $\mathrm{Mn}(6539 \mathrm{eV})$ for the sample annealed at $580{ }^{\circ} \mathrm{C}$ for $40 \mathrm{~min}$. Figure 6 (a) shows a more pronounced ASAXS effect near the absorption edge of Fe as compared to that of Mn (shown in figure 6(b)), which reveals the presence of larger amount of Fe atoms in the particles in comparison to $\mathrm{Mn}$ atoms.
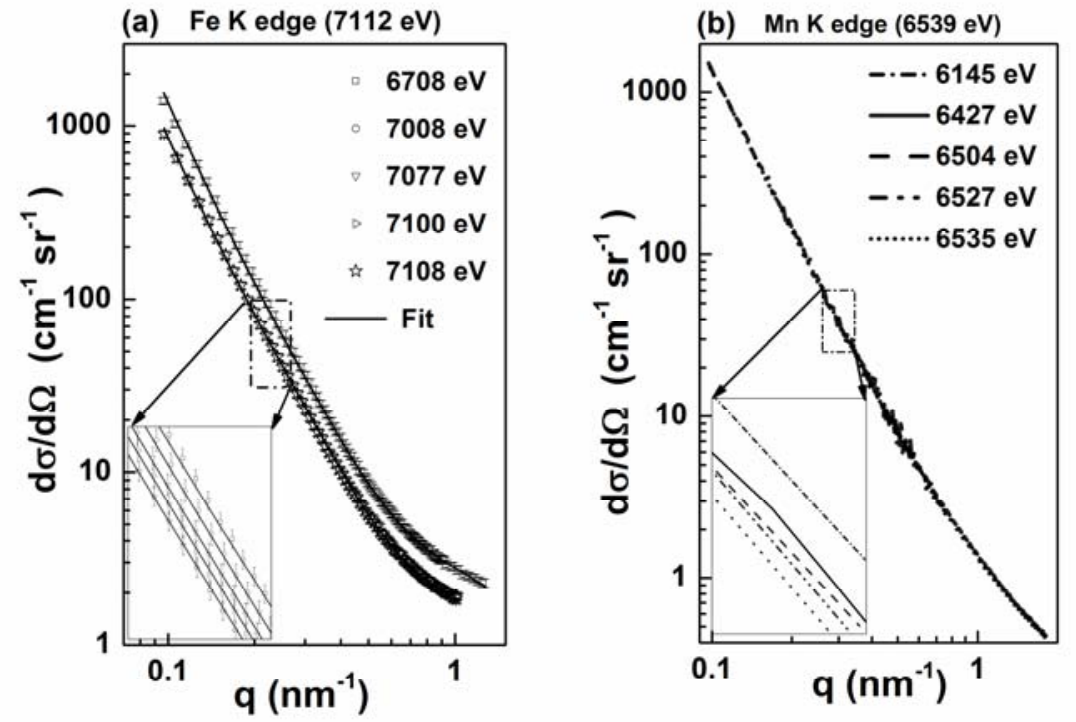

Figure 6: ASAXS scattering curves measured near the K absorption edges of Fe (a) and Mn (b). The ASAXS curves for Fe are fitted with the spherical core-shell model as shown by the solid line (a). 
The quantitative information is obtained by fitting the ASAXS curves and the experimental relative contrasts by two separate fitting routines as described in the theory section. In the first fit routine, ASAXS curves near the absorption edge of Fe fitted with the spherical core-shell model and the log normal distribution of particles as shown in Figure 6(a). For clarity in the figure, only the curves measured near and far from the absorption edge are shown, while the inset shows all five fitted curves.

In the second fit routine, the relative experimentally determined contrasts for the core and the shell are fitted simultaneously with the theoretically evaluated contrasts. Simultaneously fitted relative experimental contrasts for the core and the shell of particles near the absorption edge of Fe for the sample annealed at $580{ }^{\circ} \mathrm{C}$ for $40 \mathrm{~min}$ are shown in Figures 7(a) and 7(b). After the relative contrast fitting, the resulting parameters are shown in Table 2. Estimated parameters show density of core (particle) is $4.9 \pm 0.1 \mathrm{~g} / \mathrm{cm}^{3}$ and that of shell about $2.23 \pm$ $0.03 \mathrm{~g} / \mathrm{cm}^{3}$. The estimated density of the shell is comparable to the bulk density of $\mathrm{SiO}_{2}(2.2$ $\mathrm{g} / \mathrm{cm}^{3}$ ), which provides the first hint at an enrichment of the shell with $\mathrm{SiO}_{2}$. The evaluated parameters also reveal that the amount of Fe atoms in the particle is about $87 \%$ of the total $\mathrm{Fe}$ atoms in the system.

(a)

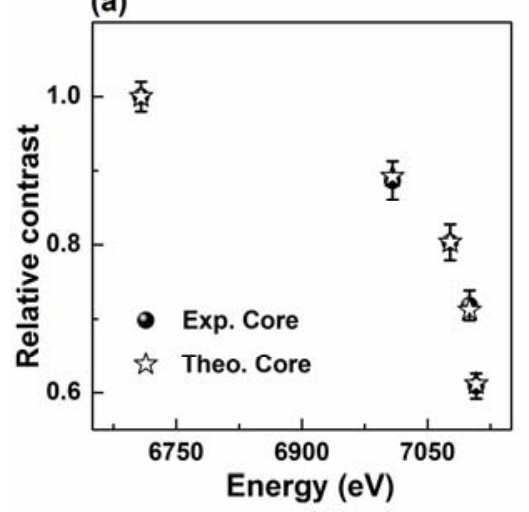

(b)

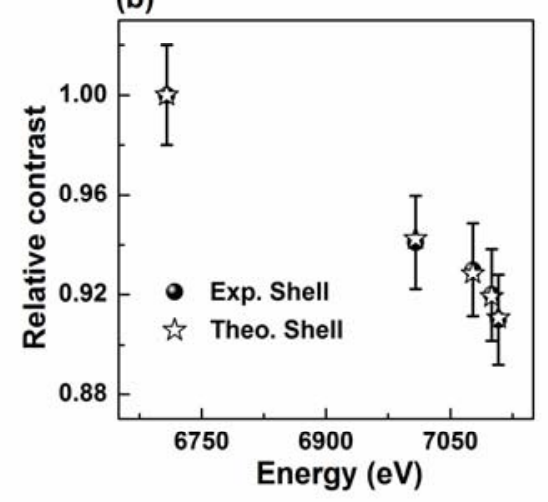

Figure 7: Comparison of the theoretical and experimental energy-dependent relative contrast for $\mathrm{Fe}(7112 \mathrm{eV})$ edge for the sample annealed at $580{ }^{\circ} \mathrm{C}$ for $40 \mathrm{~min}$. (a) Relative contrast variation for the particle (core). (b) Relative contrast variation for the shell region of the particle.

Table 2 Resulting fit parameters for the ASAXS data analysis for the sample annealed at $580{ }^{\circ} \mathrm{C}$ for $40 \mathrm{~min}$

\begin{tabular}{|c|c|}
\hline Parameters & $\begin{array}{c}\text { Sample } 580{ }^{\circ} \mathrm{C}, \\
40 \text { min }\end{array}$ \\
\hline Density core $\left(\mathrm{g} / \mathrm{cm}^{3}\right)$ & $4.9 \pm 0.1$ \\
\hline Density shell $\left(\mathrm{g} / \mathrm{cm}^{3}\right)$ & $2.23 \pm 0.03$ \\
\hline Density matrix $\left(\mathrm{g} / \mathrm{cm}^{3}\right)$ & $2.7 \pm 0.1$ \\
\hline Fe atoms in core $(\%)$ & 87 \\
\hline
\end{tabular}


Distribution of Fe atoms in the particles can be estimated by separating the pure resonant scattering contribution from the total scattering SAXS curves by using the Stuhrmann equation (6). The resonant curve for the Fe edge for the sample annealed at $580{ }^{\circ} \mathrm{C}$ for $40 \mathrm{~min}$ is shown in Figure 8(a). The curve is fitted with the spherical model and it is seen that the resulting size distribution from the resonant curve fitting is comparable to the size distribution for the core region of SANS curve as shown in Figure 8(b).

(a)

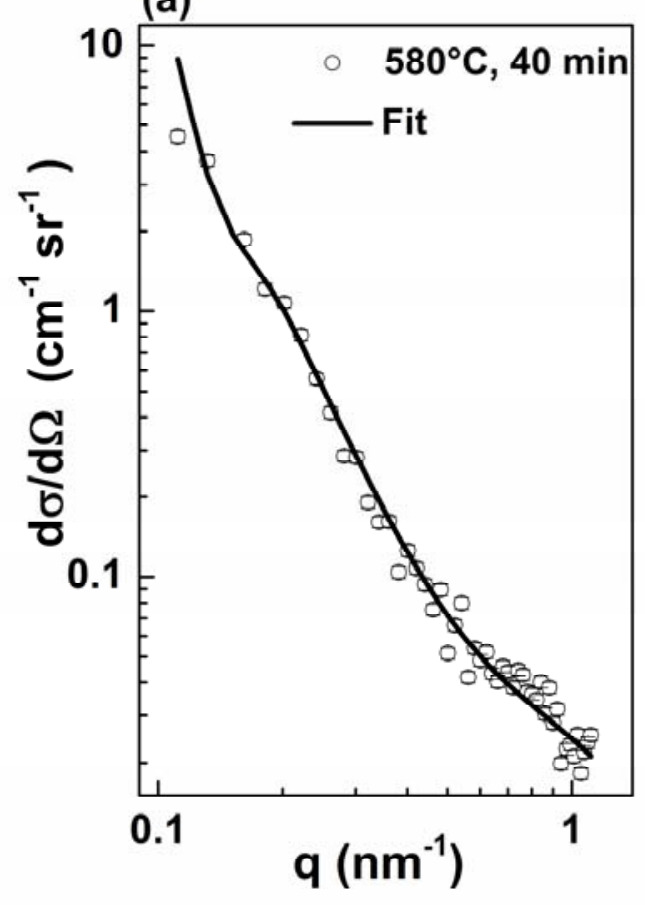

(b)

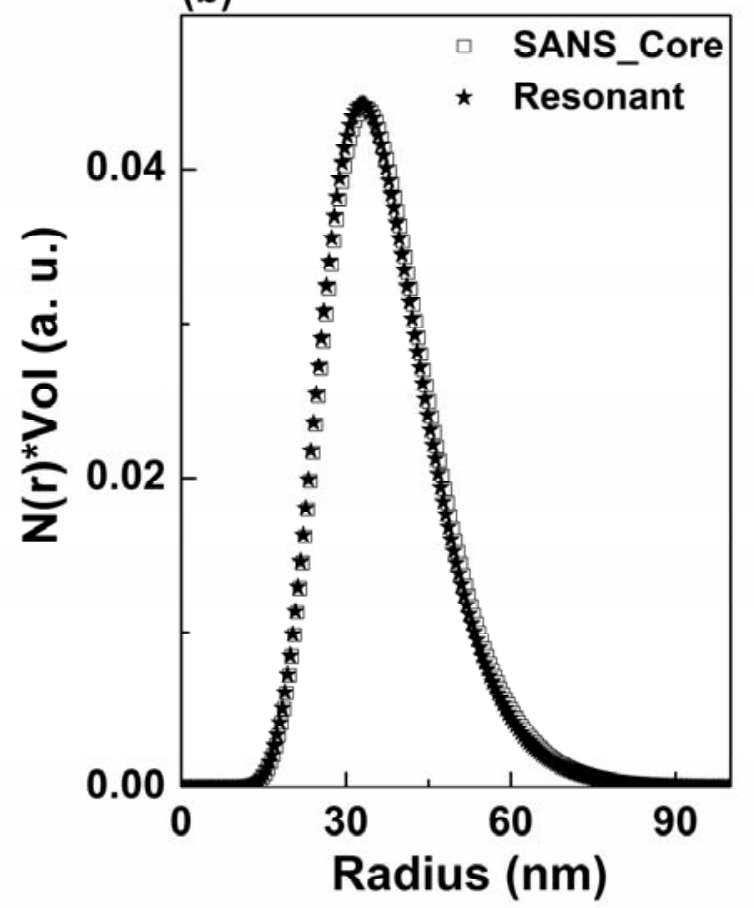

Figure 8: Resonant curves evaluated by using the Stuhrmann method for the samples heat treated at $580{ }^{\circ} \mathrm{C}$ for $40 \mathrm{~min}$. (a) Resonant curve fitted with spherical model (b) Comparison of size distributions for the core (particle) evaluated from the total scattering (SANS curve) and by the resonant curve.

\section{Discussion}

Earlier, precipitation of a magnetic (Fe-Mn-O) based spinel phase during annealing of the investigated glass ceramics was reported [35]. In the present work, samples are annealed at $580{ }^{\circ} \mathrm{C}$ for 20,40 and $60 \mathrm{~min}$. Table 1 shows the resulting parameters after SANSPOL data analyses. The results reveal that the average diameter of the particles increase from $40 \mathrm{~nm}$ to $100 \mathrm{~nm}$ for the samples annealed from $20 \mathrm{~min}$ to $60 \mathrm{~min}$. These results can be explained as follows: at higher temperature the diffusion coefficient of crystal forming elements $\mathrm{Fe}$ and $\mathrm{Mn}$ are also higher and this leads to the growth of particles with high volume fraction even when annealing for a short time of $20 \mathrm{~min}$. This observation is supported by the average sizes of the particles obtained while annealing at $550{ }^{\circ} \mathrm{C}$ for different time periods [12].

Moreover, results as presented above reveal the formation of spherical core-shell structure for the samples annealed for time intervals up to $60 \mathrm{~min}$. Here, the core represents the particle 
phase and it is surrounded by shell-like region. The spherical core-shell structure can be explained as follows: during the process of crystallization, the region surrounding the crystal is being depleted in the elements forming the core crystal (Fe and $\mathrm{Mn}$ ) and hence, enriched with the other glass components $\left(\mathrm{SiO}_{2}, \mathrm{Na}_{2} \mathrm{O}\right)$. The contrast profile shown in Figure 4 (Right) reveals that the electron density of the shell is lower than the electron density of both core and matrix. The density $\left(2.23 \pm 0.03 \mathrm{~g} / \mathrm{cm}^{3}\right)$ of the shell estimated by ASAXS is comparable to the bulk density of $\mathrm{SiO}_{2} 2.2 \mathrm{~g} / \mathrm{cm}^{3}$ and is lower than that determined for the samples with the same composition but annealed for $40 \mathrm{~min}$ at $550^{\circ} \mathrm{C}$, which is an indication of enrichment of the shell with $\mathrm{SiO}_{2}$. Actually, the shell is consisting of almost pure $\mathrm{SiO}_{2}$ in case of annealing at $580^{\circ} \mathrm{C}$. Further, it should be noted that if only Fe and Mn ions are depleted from the shell, the composition of the shell should be approximately $18 \mathrm{Na}_{2} \mathrm{O}-82 \mathrm{SiO}_{2}$, with an expected density of about $2.38 \mathrm{~g} / \mathrm{cm}^{3}$ [36]. Densities as low as $2.23 \mathrm{~g} / \mathrm{cm}^{3}$ are expected from glasses with $2-5 \mathrm{~mol} \% \mathrm{Na}_{2} \mathrm{O}$ [36]. The $\mathrm{Na}^{+}$ions do not penetrate the growing spinel nanocrystals. Therefore, the sodium ions, depleted from the shell, are remaining in the glass matrix. A tendency towards phase separation in binary sodium silicate glasses and melts with the formation of silica-rich inclusions in the sodium silicate matrix is reported in Ref. [37]. In our case, the depletion of sodium ions from the shell is additionally supported by the higher concentration of $\mathrm{Fe}^{3+}$ ions in the glass matrix where the $\mathrm{Na}^{+}$ions are serving for charge compensation of the tetrahedrally coordinated $\mathrm{Fe}^{3+}$ ions. The latter idea is supported by the even higher matrix density of $2.7 \mathrm{~g} / \mathrm{cm}^{3}$ reported here in comparison to the matrix density estimated for the samples annealed for the same time period but at $550^{\circ} \mathrm{C}-2.39 \mathrm{~g} / \mathrm{cm}^{3}$ [12]. In both cases, the formation of $\mathrm{Mn}_{\mathrm{x}} \mathrm{Fe}_{3-\mathrm{x}} \mathrm{O}_{4}$ spinel type of magnetic nanocrystals surrounded by a shell enriched in $\mathrm{SiO}_{2}$ along with the other glass components is suggested by ASAXS [12]. Such type of crystallization process having an $\mathrm{Si}$ enriched layer surrounding the fluoride particles was reported earlier $[22,38,39,45]$. Here the viscosity of the surrounding layer plays an important role in the growth of the particles. When the viscosity of the layer is lower than that of the bulk, this leads to an increase in the crystal growth. When the viscosity of the layer increases, the layer acts as a barrier and decelerates the crystal growth as reported earlier [40$42,44]$. The glass transition temperatures of binary sodium silicate glasses containing $2-5$ mol \% of $\mathrm{Na}_{2} \mathrm{O}$, which is the supposed composition of the shell, are between 500 and $510{ }^{\circ} \mathrm{C}$ [36]. This is well below the crystallization temperature of $580{ }^{\circ} \mathrm{C}$. Therefore, at $580{ }^{\circ} \mathrm{C}$ the shell does not act as a kinetic barrier for the diffusion of iron and manganese ions towards the growing crystal. Accordingly, at this crystallization temperature the thermodynamic factors are more important for the crystallization kinetics than the kinetic ones. 


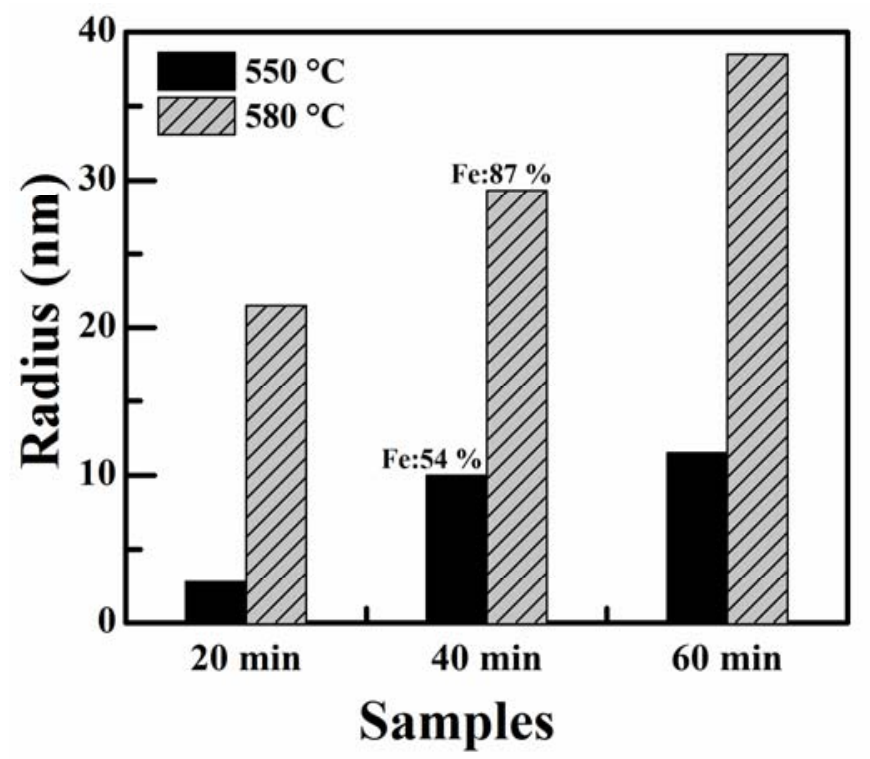

Figure 9: Comparison of particle size obtained by SANSPOL data analysis for the sample heat treated at $550^{\circ} \mathrm{C}$ and $580^{\circ} \mathrm{C}$. Fe concentration in the nanoparticles evaluated by ASAXS for the sample annealed for $40 \mathrm{~min}$ at $550{ }^{\circ} \mathrm{C}$ and $580{ }^{\circ} \mathrm{C}$ is also shown.

Figure 9 shows the comparison of particle size obtained by SANSPOL data analyses for the sample heat treated at $550{ }^{\circ} \mathrm{C}$ and $580{ }^{\circ} \mathrm{C}$.

ASAXS analysis for the sample annealed for 40 min shows the particles are highly enriched in Fe atoms (about $87 \%$ ), which is also proved by analysis of the resonant curve shown in Figure 8. As expected, this number is larger, than those reported earlier for a sample annealed at $550{ }^{\circ} \mathrm{C}$, namely $54 \%$ for 40 minutes and up to $84 \%$ for 180 minutes [12].

Furthermore, SANSPOL investigations for the sample annealed for 20, 40 and 60 min show that both core and shell give rise to nuclear scattering while only the core produces magnetic scattering. The size of the sphere fitting the magnetic scattering coincides with the size of the core of the nuclear scattering as shown in Table 1. Moreover, Figure 9 shows the particle size comparison obtained by SANSPOL data analyses for the samples heat treated at 550 and $580^{\circ} \mathrm{C}$ [43]. It should also be noted that for both annealing temperatures the shortest annealing time leads to the smallest magnetic particles but the next longer annealing period always results in much larger magnetic particles, especially for annealing at $550^{\circ} \mathrm{C}$. The longer annealing times always lead to average size of the particles slowly increasing with increasing annealing time. This effect is more pronounced for the lower annealing temperature of 550 ${ }^{\circ} \mathrm{C}$, as seen in Figure 9 and Table 1 and could be explained by the higher viscosity of the glass matrix at lower temperatures which impedes the diffusion of the $\mathrm{Fe}$ and $\mathrm{Mn}$ ions towards the growing magnetic crystals. The same tendency is also observed with respect to the increase in the thickness of the formed shells. The results show that for both annealing temperatures, the presence of $\mathrm{Fe}$ in the particles core gives rise to the strong magnetic properties while the shell remains weakly magnetic. This confirms the ASAXS conclusion, that the shell is a diffusion 
zone depleted in Fe and Mn. The size distributions for the samples evaluated by SANSPOL measurements are comparable with those obtained by the ASAXS resonant curve investigations as shown in Figure 8(b), which reveal that the results from the two types of small angle scattering investigations agree completely.

\section{Conclusion}

In conclusion, we have investigated magnetic nanoparticles embedded in silicate glass samples annealed at $580{ }^{\circ} \mathrm{C}$ for different time periods by two small angle scttering methods, SANSPOL and ASAXS. Data analyses show the formation of nanoparticles with spherical core-shell structures for the samples annealed for 20, 40 and $60 \mathrm{~min}$. Results show the growth of particles from $40 \mathrm{~nm}$ to $100 \mathrm{~nm}$ in diameter and thickness of the shell from $1.4 \mathrm{~nm}$ to 2.5 $\mathrm{nm}$ with increasing annealing times. ASAXS investigations near the Fe and Mn absorption edge show that the particles are highly enriched in Fe atoms, but the layer surrounding the particles (shell) is mainly enriched with $\mathrm{SiO}_{2}$ and probably the other constituents of the glass. SANSPOL investigation reveals that the size of the structural entities with strong magnetic properties coincides with the cores determined by ASAXS while the shell surrounding them has weak magnetic properties. Evaluated results from SAS measurement with X-rays and neutrons are well comparable to each other.

\section{Acknowledgement}

The authors would like to acknowledge Dr. Ivailo Gugov (University of Chemical Technology and Metallurgy, Sofia, Bulgaria) and Prof. Dr. Klaus Rademann (HumboldtUniversität zu Berlin) for discussions on the manuscript. Finally the authors would like to acknowledge Dr. Charles Dewhurst (ILL, Grenoble, France) for the SANSPOL measurements.

Figure $12 \mathrm{D}$ scattering intensity patterns from the polarized SANS experiment after data correction for the sample annealed at $580{ }^{\circ} \mathrm{C}$ for $40 \mathrm{~min}$. The magnetic field of 1.5 Tesla is applied in horizontal direction perpendicular to the incident neutron beam. Intensities are measured under different polarization states of incident neutrons antiparallel $(\mathrm{ON})$ to the magnetic field, parallel (OFF) to the magnetic field, and (ON - OFF) is the difference term of $(\mathrm{ON})$ and $(\mathrm{OFF})$.

Figure 2 Separated nuclear and magnetic SANS curves for the sample annealed at $580{ }^{\circ} \mathrm{C}$ for $40 \mathrm{~min}$ are shown. The nuclear curve is fitted with the spherical core-shell model and the magnetic curve is fitted with spherical model. Solid line shows the fit. 
Figure 3 Nuclear and magnetic scattering curves for the sample annealed at $580{ }^{\circ} \mathrm{C}$ for different time periods. (a) Nuclear scattering curves are fitted with the spherical core shell model. (b) Magnetic scattering curves are fitted with the spherical model. Solid line shows the fit.

Figure 4 Sketch of the spherical core-shell model (left). Relative contrast profile to fit the SAXS, ASAXS and SANS scattering curves (right).

Figure 5 Comparison of size distributions for the SAXS and SANS (magnetic and nuclear) scattering estimated by fitting the scattering curves.

Figure 6 ASAXS scattering curves measured near the K absorption edges of Fe (a) and Mn (b). The ASAXS curves for Fe are fitted with the spherical core-shell model as shown by the solid line (a).

Figure 7 Comparison of the theoretical and experimental energy-dependent relative contrast for both $\mathrm{Fe}(7112 \mathrm{eV})$ and $\mathrm{Mn}(6539 \mathrm{eV})$ edges for the sample annealed at $580{ }^{\circ} \mathrm{C}$ for $40 \mathrm{~min}$. (a) Relative contrast variation for the particle (core). (b) Relative contrast variation for the shell region of the particle.

Figure 8 Resonant curves evaluated by using the Stuhrmann method for the samples heat treated at $580{ }^{\circ} \mathrm{C}$ for $40 \mathrm{~min}$. (a) Resonant curve fitted with spherical model (b) Comparison of size distributions for the core (particle) evaluated from the total scattering (SAXS curve) and by the resonant curve.

Figure 9 Comparison of particle size obtained by SANSPOL data analysis for the sample heat treated at $550{ }^{\circ} \mathrm{C}$ and $580{ }^{\circ} \mathrm{C}$. Fe concentration in the nanoparticles evaluated by ASAXS for the sample annealed for $40 \mathrm{~min}$ at $550{ }^{\circ} \mathrm{C}$ and $580{ }^{\circ} \mathrm{C}$ is also shown.

\section{References}

[1] Coey JMD (1999) Whither magnetic materials? J. Magn. Magn. Mater. 196: 1-7

[2] Shull RD, Bennett LH (1992) Nanocomposite magnetic materials. Nanostruc. Mater. 1: 83-88

[3] Owen AE, Spear WE (1976) Electronic properties and localised states in amorphous semiconductor. Phys. Chem. Glas. 17: 174-192

[4] Siegel RW (1993) Nanostructured materials -mind over matter- Nanostruc. Mater. 3: 1-18

[5] Hoell A, Wiedenmann A, Heyen U, Schüler D (2004) Nanostructure and field-induced arrangement of magnetosomes studied by SANSPOL. Physica B 350: 309-313

[6] Odenbach S (2004) Recent progress in magnetic fluid research. J. Phys. Cond. Mat. 16: 11351150

[7] Aytur T, Foley J, Anwar M, Boser B, Harris E, Beatty PR (2006) A novel magnetic bead bioassay platform using a microchip-based sensor for infectious disease diagnosis. J. Imm. Meth. 314: 21-29

[8] Sakai Y, Abe N, Takeuchi S, Takahashi F (1995) BOD Sensor Using Magnetic Activated Sludge. J Ferment Bioeng. 80: 300-303

[9] Carta D, Casula MF, Mountjoy G, Corrias A (2008) Formation and cation distribution in supported manganese ferrite nanoparticles: an X-ray absorption study. Phys. Chem. Chem. 
Phys. 10: 3108-3117

[10] Harizanova R, Völksch G, Rüssel C (2010) Microstructures formed during devitrification of $\mathrm{Na} 2 \mathrm{O}$ A12O3 B2O3 SiO2 Fe2O3 glasses. J. Mater. Sci. 45: 1350-1353

[11] Harizanova R, Völksch G, Rüssel C (2011) Crystallization and microstructure of glasses in the system Na2O/MnO/SiO2/Fe2O3. Mater. Res. Bull. 46: 81-86

[12] Raghuwanshi VS, Tatchev T, Harizanova R, Haas S, Hoell A, Gugov I, Rüssel C (2012) Structural analysis of magnetic nanocrystals embedded in silicate glasses by anomalous smallangle X-ray scattering. J. App. Cryst. 45: 644-651

[13] Dewhurst C 2003 http://www.ill.eu/?id=6304

[14] Hoell A, Zizak I, Bieder H, Mokrani L (2006) DE Patent Specification 102006029449

[15] Feigin LA, Svergun DI (1987) Structure analysis by Small angle X-ray and Neutron Scattering (New York: Plenum Press)

[16] Glatter O, Kratky O (1982) Small-Angle X-ray Scattering (New York: Academic Press)

[17] Guinier A, Fournet G (1955) Small angle Scattering of X ray (New York: Wiley)

[18] Pederson JS (1997) Analysis of small-angle scattering data from colloids and polymer solutions: modeling and least-squares fitting. Adv. Coll. Int. Sci. 70: 171-210

[19] Pederson JS (2002) Modelling angle scattering of small- data from colloids and polymer systems, in Neutrons, X-rays and Light: Scattering Methods Applied to Soft Condensed Matter (Lindner P. and Zemb Th., eds), pp. 391-420. Elsevier Science B.V., Amsterdam. Amsterdam:Elsevier 391-420

[20] Vrij A (1979) Mixtures of hard spheres in the Percus-Yevick approximation. Light scattering at finite angles. J. Chem. Phys. 71: 3267-3270

[21] Haas S, Hoell A, Wurth R, Rüssel C, Boesecke P, Vainio U (2010) Analysis of nanostructure and nanochemistry by ASAXS: Accessing phase composition of oxyfluoride glass ceramics doped with Er3+ ÕYb3+. Phys. Rev. B 81: 184207

[22] Raghuwanshi VS, Bocker C, Hoell A, Rüssel C (2012) Experimental evidence of a diffusion barrier around BaF2 nanocrystals in a silicate glass system by ASAXS. CrystEngComm. 14: $5215-5223$

[23] Cromer DT, Liberman D (1970) Relativistic Calculation of Anomalous Scattering Factors for X Rays. J. Chem. Phys. 53: 1891-1898

[24] Kohlbrecher J and Bressler I SASfit, Software package for fitting small-angle scattering curves. http://kur.web.psi.ch/sans1/SANSSoft/sasfit.html

[25] Hoell A, Tatchev D, Haas S, Haug J, Boesecke P (2009) On the determination of partial structure functions in small-angle scattering exemplified by A189Ni6La5 alloy. J. App. Cryst. 42: $323-325$

[26] Stuhrmann H B (1985) Resonance Scattering in Macromolecular Structure Research Adv. Poly. Sci. 67: 123-163

[27] Kammel M, Hoell A, Wiedenmann A (2001) Structure of Magnetite Ferrofluids investigated by SANS with polarized neutrons. Scripta Mater. 44: 2341-2345

[28] Kammel M, Wiedenmann A, Hoell A (2002) Nuclear and magnetic nanostructure of 
magnetite ferrofluids studied by SANSPOL J. Magn. Magn. Mater. 252: 89-91

[29] Kohlbrecher J, Wiedenmann A, Wollenberger H (1997) Magnetic coupling between the different phases in nanocrystalline Fe-Si-B studied by small angle neutron scattering. Z. Phys. B. 104: 1-4

[30] Wiedenmann A, Hoell A, M Kammel (2002) Small-angle scattering investigations of cobaltferrofluids using polarised neutrons. J. Magn. Magn. Mater. 252: 83-85

[31] Wiedenmann A, Kammel M, Heinemann A, Keiderling U (2006) Nanostructures and ordering phenomena in ferrofluids investigated using polarized small angle neutron scattering. J. Phys. Cond. Mat. 18: S2713-S2736.

[32] Tatchev D, Hoell A, Kranold R, Armyanov S (2005) Size distribution and composition ofmagnetic precipitates in amorphous Ni-P alloy. Physica B 369: 8-19

[33] Wiedenmann A (2000) Small-angle neutron scattering investigations of magnetic nanostructures using polarized neutrons. J. App. Cryst. 33: 428-432

[34] Haas S (2010) Nanochemische Zusammensetzungsanalyse mittels anomaler Röntgenkleinwinkelstreuung (ASAXS): Erbium und Ytterbium dotierte OxyfluoridGlaskeramiken, (Humboldt University of Berlin, Germany)

[35] Harizanova R, Gugov I, Rüssel C, Tatchev D, Raghuwanshi VS, Hoell A (2011) Crystallization of (Fe, Mn)-based nanoparticles in sodium-silicate glasses. J. Mat. Sci. 46: $7169-7176$

[36] Knoche R, Dingwell DB, Seifert DA, Webb SL (1994) Non-linear properties of supercooled liquids in the system Na20-SiO2. Chem. Geo.116: 1-16

[37] Jarry P, Richet P (2001) Unmixing in sodium-silicate melts: influence on viscosity and heat capacity. J. Non-Cryst. Sol. 293: 232-237

[38] Rüssel C (2005) Nano-crystallization of $\mathrm{CaF} 2$ from Na2O/K2O/CaO/CaF2/A12O3/SiO2. Chem. Mater. 17: 5843-5847

[39] Bocker C, Rüssel C (2009) Self-organized nano-crystallisation of BaF2 from $\mathrm{Na} 2 \mathrm{O} / \mathrm{K} 2 \mathrm{O} / \mathrm{BaF} 2 / \mathrm{A} 12 \mathrm{O} 3 / \mathrm{SiO} 2$ glasses. J. Eur. Cer. Soc. 29: 1221-1225

[40] Avramov I, Rüssel C, Kolkovska N, Georgiev I (2008) Crystallization kinetics and network rigidity. J. Phys.: Cond. Mat. 20: 335203

[41] Bhattacharya S, Bocker C, Heil T, Jinschek JR, Höche T, Rüssel C, Kohl H (2009) Experimental Evidence of Self-Limited Growth of Nanocrystals in Glass. Nano Lett. 9: 24932496

[42] Bocker C, Bhattacharya S, Rüssel C (2009) Size distribution of BaF2 nanocrystallites in transparent glass ceramics. Acta Mater. 57: 5956-5963

[43] Raghuwanshi VS, Harizanova R, Haas S, Tatchev D, Gugov I, Dewhurst C, Rüssel C, Hoell A (2014) Magnetic nanocrystals embedded in silicate glasses studied by polarized SANS. J. Non-Cryst. Sol. 385: 24-29

[44] Raghuwanshi VS, Rüssel C, Hoell A (2014) Crystallization of ZrTiO4 Nanocrystals in Lithium-Alumino-Silicate Glass Ceramics: Anomalous Small-Angle X ray Scattering Investigation. Cryst. Growth \& Des. 14: 2838-2845 
[45] Hoell A, Varga Z, Raghuwanshi VS, Krumrey M, Bocker C, Russel C (2014) ASAXS study of $\mathrm{CaF} 2$ nanoparticles embedded in a silicate glass matrix. J. App. Cryst. 47: 60-66 